\title{
Forensic DNA methylation profiling from evidence material for investigative leads
}

\author{
Hwan Young Lee ${ }^{1, *}$, Soong Deok Lee ${ }^{2}$ E Kyoung-Jin Shin ${ }^{1}$ \\ ${ }^{1}$ Department of Forensic Medicine, Yonsei University College of Medicine, Seoul 03722, ${ }^{2}$ Department of Forensic Medicine, Seoul National \\ University College of Medicine, Seoul 03080, Korea
}

DNA methylation is emerging as an attractive marker providing investigative leads to solve crimes in forensic genetics. The identification of body fluids that utilizes tissue-specific DNA methylation can contribute to solving crimes by predicting activity related to the evidence material. The age estimation based on DNA methylation is expected to reduce the number of potential suspects, when the DNA profile from the evidence does not match with any known person, including those stored in the forensic database. Moreover, the variation in DNA implicates environmental exposure, such as cigarette smoking and alcohol consumption, thereby suggesting the possibility to be used as a marker for predicting the lifestyle of potential suspect. In this review, we describe recent advances in our understanding of DNA methylation variations and the utility of DNA methylation as a forensic marker for advanced investigative leads from evidence materials. [BMB Reports 2016; 49(7): 359-369]

\section{INTRODUCTION}

In recent years, forensic DNA typing mainly focuses on matching a suspect with the evidence by testing a set of short tandem repeat (STR) markers. With advances in the DNA detection technology and the increased number of available markers, useful DNA profiles can be obtained from highly damaged evidence materials. However, DNA profiles can often fail to identify persons when there is no suspect available, and the evidence DNA profile does not match that of any person in the forensic DNA database $(1,2)$. When no other evidence is available, hundreds to thousands of volunteers can be invited to provide their samples for DNA mass screening; however, such DNA dragnets without specific

${ }^{*}$ Corresponding author. Tel: +82-2-2228-2482; Fax: +82-2-3620860; E-mail: hylee192@yuhs.ac

http://dx.doi.org/10.5483/BMBRep.2016.49.7.070

Received 20 April 2016

Keywords: Age prediction, Body fluid identification, DNA methylation, Forensic genetics, Investigative lead cause and evidence to ask volunteers often face criticisms and are legally forbidden in some countries because of ethical concerns $(1,3)$.

Nowadays, forensic phenotyping, aiming to infer the unknown sample donor's appearance from DNA, is expected to reduce the number of potential suspects $(4,5)$. To date, the most well studied externally visible characteristic (EVC) markers are the single nucleotide polymorphism (SNP) markers associated with pigmentation, e. g., the variations in the coloration of the iris, hair, and skin (6-8). However, such pigmentation markers may not be useful in certain populations such as Asians and Africans with little color variation. More recently, age has been suggested as an EVC that can be used regardless of the ethnicity to predict an individual's appearance, thereby providing an investigative lead to track an unknown suspect or identify a missing person. Recently, a DNA test based on sjTREC DNA quantification has been introduced in the forensic field, and is based on the existing knowledge of the decrease in T-cells and a particular T-cell DNA rearrangement in blood with increased age (9). This test has shown relatively high prediction accuracy with an error of 9 years. However, this method cannot be applied to the age prediction of other body fluids that do not include T-cells and needs to be tested using patients' blood samples because of its immunedependent characteristics. In the meantime, the advancement of epigenetics led to the identification of a number of CpG markers, exhibiting age-associated DNA methylation changes in various types of tissues and cells. Several studies have reported age-predictive models that could be applied across a broad spectrum of tissues as well as those based on the use of blood or saliva with considerable prediction accuracy $(<5$ years) (10-13). To date, DNA methylation is regarded as the most promising age-predictive biomarker.

In situations where the evidence profile matches a suspect's DNA, the suspect is not always proved guilty, because the matching DNA profiles only help addressing the issue at source or sub-source level. The probative force of matching DNA profile for sub-source and source level issues, in most cases, does not transfer directly to the probative force at activity or offence level (14). Therefore, the activity leading to the deposition of the evidence material is mainly assessed by forensic experts $(15,16)$; offence level issues are normally 
dealt with by the court. To evaluate the evidence at the activity level, knowledge about the cell types in evidence material as well as the investigation of fingerprints and bloodstain pattern analysis will be helpful (17). For example, the detection of semen from vaginal swabs can indicate the involvement of some form of sexual encounter or assault, and blood stains can indicate some form of physical struggle, assault or murder, whereas menses may be proposed as an alternative inoffensive scenario for a blood stain in an alleged violent assault (17). In the past decades, numerous types of analytical methods including chemical tests, immunological tests, protein catalytic activity tests, spectroscopic methods, and microscopy have been developed for the investigation of forensically relevant body fluids (18). However, most of these methods have limitations such as low specificity, lack of sensitivity, sample destruction, instability of biomolecule assayed, or incompatibility with downstream STR analysis (19). In recent years, molecular approaches detecting specific messenger RNA (mRNA) and micro RNA (miRNA) expressions and differential DNA methylation patterns have therefore been intensively investigated (17). Among these methods, DNA-methylation based assays identifying differential DNA methylation profiles of different cells or tissue types have been proposed for distinguishing different types of body fluids owing to their high specificity, DNA-based testing characteristics, fit with the current forensic casework application, and applicability to old cases with only DNA extracts available.

DNA methylation has only recently come into focus in the forensic field; however, its applicability is being highly estimated among an increasing number of forensic investigators. Just like epigenetics to genetics, DNA methylation analyses are now expected to add more informative layers to the forensic genetic analyses of the evidence materials.

\section{BIOLOGICAL BACKGROUND OF DNA METHYLATION}

\section{DNA methylation}

Methylation of nucleotides provides a molecular mean to reversibly mark genomic DNA (20). DNA methylation is involved in immune recognition in bacteria, but regulates the structure and expression of the genome in complex higher eukaryotes (21). In eukaryotes, methylation only occurs at the cytosine residue and is different from those in bacteria that can methylate adenosine or cytosine residue (20). Moreover, vertebrates are unique as cytosine methylation occurs throughout the entire genome, whereas plants and invertebrates show mosaic methylation patterns with only specific genomic elements targeted (20).

Despite the widespread methylation in vertebrate genomes, CpG islands (CGIs), which often overlap with promoter regions, generally remain unmethylated, whereas CG-poor promoters are methylated when not active (20). Because DNA methylation blocks the start of transcription initiation not elongation (22), CGI methylation at the transcription start site is also associated with long-term silencing, e. g., X-chromosome inactivation, imprinting, and genes expressed dominantly in germ cells and some tissue-specific genes (22). In contrast, sometimes, CGls in gene bodies are known to be methylated in a tissue-specific manner, and non-CGI methylation is more dynamic and more tissue-specific than the CGI methylation (22).

Such pattern of DNA methylation is established by de novo DNA methyltransferases DNMT3A and DNMT3B in combination with DNMT3L and is faithfully maintained through cell division by maintaining methyltransferase DNMT1 and associated proteins (23). DNA methylation has long been considered to be lost passively through imperfect maintenance, but the recent discovery of mammalian ten-eleven translocation family of proteins showed a convincing path for catalyzed active demethylation (24).

\section{DNA methylation variations}

DNA methylation at a single $\mathrm{CpG}$ site within a single DNA strand is a binary trait, as the site is either methylated or not (25). However, experimental samples contain a large number of DNA strands, and therefore DNA methylation, which is recorded as a fraction between zero and one, represents the frequency of methylation at a given $\mathrm{CpG}$ site across the population of cells in the sample (11).

DNA methylation changes during the development and aging. The full range of DNA methylation variation is potentially enormous considering that the diploid human genome contains $\geq 10^{7} \mathrm{CpGs}$ that may all potentially vary (26). The most common features in which DNA methylation varies appear as methylation variable position (MVP), variably methylated region (VMR) or differentially methylated region (DMR) (27-29). DNA methylation at a single CpG site is known as a MVP, and the region defined by increased variability rather than gain or loss of DNA methylation is referred as VMR. DMR is a region of the genome at which multiple adjacent $\mathrm{CpG}$ sites show differential methylation and can occur in many different contexts, e. g., imprinting-specific DMR (iDMR), tissue-specific DMR (tDMR), reprogrammingspecific DMR (rDMR), cancer-specific DMR (CDMR), and aging-specific DMR (aDMR).

The factors underlying variable DNA methylation include cell differentiation, aging, and environmental exposures and genetic factors. During early embryo development, cell-specific DNA methylation patterns develop to aid in cell differentiation, thereby enabling cells to have specific structures and functions (30). The established DNA methylation patterns are maintained through consecutive cell divisions, and are relatively stable throughout life (25). However, aging can modify DNA methylation through epigenetic drift and epigenetic clock; epigenetic drift is caused by the stochastic accumulation of small errors in transmitting and maintaining DNA methylation (31), and epigenetic clock refers to the phenomenon in which specific sites in the genome undergo DNA methylation changes with age that are progressive and 
common across individuals and sometimes even tissues (11, 12). In addition, environmental exposures such as diet, stress, or smoking can alter DNA methylation at various stages of human development (25). Besides, twin and family-based studies suggested that a considerable amount of inter-individual variation in DNA methylation is in part determined genetically $(32,33)$. The genome-wide association studies, which test genotype-phenotype associations, identified a large number of SNPs associated with the DNA methylation levels at various CpGs $(34,35)$. Therefore, DNA methylation at a certain CpG site is the result of multiple processes that are regulated both by genetic and environmental factors.

\section{FORENSIC APPLICATIONS OF DNA METHYLATION}

\section{Tissue-specific DNA methylation changes and forensic body fluid identification}

A significant number of studies have reported tissue-specific DNA methylation and gene expression variations $(17,18)$. In fact, tissue of origin is the primary difference in DNA methylation profiles from different samples, regardless of their origin from the same or different individuals (36-39). Genome-wide DNA methylation analysis of different tissues demonstrated that numerous tDMRs exist in the mammalian genome, and DNA methylation patterns were more consistent among the same tissues from different individuals than among different tissues from the same individual (36).

In the forensic field, the potential of tissue-specific differential DNA methylation has been examined for the identification of body fluids that are frequently observed in crime scenes (40-45). Frumkin et al. (40) first reported genomic loci that are differentially methylated among blood, saliva, semen, skin epidermis, urine, and vaginal secretion using a method based on methylation-sensitive restriction enzyme-PCR (MSRE-PCR). Lee et al. (41) examined the potential of tDMRs for forensic body fluid identification by the bisulfite sequencing method. They suggested two testis-specific tDMRs located at the DACT1 and USP49 genes as a semen-specific marker and the PFN3 tDMR as a vaginal fluid-specific marker. Madi et al. (42) also examined a few genomic loci using bisulfite modification and pyrosequencing and reported that the methylation patterns at the ZC3H12D and FGF7 loci can differentiate sperm from other biological samples, whereas the C20orf117 locus and the BCAS4 locus can differentiate blood and saliva from other samples, respectively.

Recently, rapid advances in epigenetics have made genome-wide DNA methylation profiling accessible to many researchers. In particular, as Illumina's HumanMethylation450 (450K) BeadChip array provides DNA methylation profiles at $\geq 450,000 \mathrm{CpG}$ sites using only as little as $0.5 \mu \mathrm{g}$ of genomic DNA, several researchers have reported $\mathrm{CpG}$ markers that show differential DNA methylation patterns in different types of body fluids based on the $450 \mathrm{~K}$ BeadChip array results. Park et al. (43) investigated the methylome data from 16 samples of blood, saliva, semen, and vaginal secretions and identified eight CpG sites as forensically relevant DNA methylation markers: cg06379435 and cg08792630 for blood; cg26107890 and cg20691722 for saliva; cg23521140 and cg17610929 for semen; and cg01774894 and cg14991487 for vaginal secretions. In a subsequent validation with pyrosequencing analysis, the blood and semen markers were confirmed to have high specificity for the identification of the target body fluid; however, their vaginal secretion and saliva markers did not show a sufficient methylation difference from other body fluids for acceptable specificity. Lee et al. (44) also identified markers using the same approach of generating the $450 \mathrm{~K}$ BeadChip array data for 42 body fluids including blood, saliva, semen, vaginal fluid, and menstrual blood. Further validation with bisulfite sequencing and methylation SNaPshot produced a total of 8 CpGs located at or adjacent to the sites investigated in the BeadChip array as body fluid-specific markers: cg17610929, cg26763284, and cg17621389 for semen; cg06379435 and cg01543184 for blood; cg09765089-231d (a CpG located 231 bp downstream of cg09765089) and cg26079753-7d (a CpG located 7 bp downstream of cg26079753) for vaginal fluid; and cg09652652-2d (a CpG located 2 bp downstream of cg09652652) for saliva. From both of the two studies, cg17610929 and cg06379435 have been suggested as a semen-specific marker and a blood-specific marker, respectively. In addition, cg08792630 that had been proposed as a blood-specific marker by Park et al. was confirmed to show methylation signal only in blood even when examined using the array data generated by Lee et al. (unpublished data). More recently, Forat et al. (45) selected body fluid-specific CpG marker candidates from HumanMethylation27 (27K) and 450K BeadChip array data of pooled DNA samples from various body fluids and tissues: cg26285698 and cg03363565 for blood; cg09696411 for menstrual blood; cg21597595 and cg15227982 for saliva; and cg14991487 and cg03874199 for vaginal fluid; and cg22407458 and cg05656364 for semen. Through further validation with methylation SNaPshot called methylation-sensitive single nucleotide primer extension (Ms-SNuPE), they suggested body fluid-specific CpG markers that were not identical with those investigated by the BeadChip array but were adjacent to them with 0 to $288 \mathrm{bp}$ apart. However, no marker overlaps were observed between Forat et al. (45) and the two previous studies, suggesting the need to further evaluate those markers.

Nevertheless, because body fluid-specific hypo- or hypermethylation status, which does not provide "on or off" signal, has attracted criticism when used for the analysis of mixed samples, it is notable that the three recent studies (43-45) reported a set of $\mathrm{CpG}$ markers that show a methylation signal only in the target body fluids through genome-wide methylation profiling and gene-specific confirmation. Overall, the identification and use of semen-specific $\mathrm{CpG}$ markers is not a problem because of the significant difference in DNA methylation between somatic and germ cells. However, 
because most somatic tissues and body fluids possess similar DNA methylation patterns across the genome, CpGs that have been suggested to be specific to blood, saliva, vaginal fluid, and menstrual blood should be carefully validated for better applications to forensic caseworks.

\section{Age-associated DNA methylation changes and forensic age estimation}

Age-associated DNA methylation changes have been extensively studied both globally and at specific regions (46). These studies indicated a gain in DNA methylation in early life

Table 1. DNA methylation-based age predictive models

\begin{tabular}{|c|c|c|c|c|c|}
\hline Models & Age signatures $^{\mathrm{a}}$ & Gene & Tissue & Analysis platform & Accuracy $^{b}$ \\
\hline \multirow[t]{3}{*}{ Bocklandt et al. (10) } & cg09809672 & EDARADD & Saliva & $27 \mathrm{~K}$ array & 5.2 years \\
\hline & cg27210390 & TOM1L1 & & & \\
\hline & cg12799895 & NPTX2 & & & \\
\hline \multirow[t]{5}{*}{ Koch \& Wagner (49) } & cg12799895 & NPTX2 & Various tissues & $27 \mathrm{~K}$ array & 11 years \\
\hline & cg07533148 & TRIM58 & & & \\
\hline & cg25148589 & GRIA2 & & & \\
\hline & cg01530101 & KCNQ1DN & & & \\
\hline & cg23571875 & BIRC4BP & & & \\
\hline Hannum et al. (11) & $71 \mathrm{CpG}$ sites & & Blood & $450 K$ array & 3.9 years $^{c}$ \\
\hline Horvath (12) & 353 CpG sites & & Various tissues & $27 \mathrm{~K} \& 450 \mathrm{~K}$ array & 3.6 years $^{c}$ \\
\hline \multirow[t]{3}{*}{ Weidner et al. (13) } & cg25809905 & ITGA2B & Blood & Pyrosequencing & 4.3 years \\
\hline & cg02228185 & ASPA & & & \\
\hline & $\operatorname{cg} 17861230(-14$ bp) & PDE4C & & & \\
\hline \multirow[t]{2}{*}{ Zbieć-Piekarska et al. (55) } & cg16867657 (-2 bp) & ELOVL2 & Blood & Pyrosequencing & 5.0 years \\
\hline & $\operatorname{cg} 16867657(-10 \mathrm{bp})$ & & & & \\
\hline \multirow[t]{5}{*}{ Zbieć-Piekarska et al. (56) } & $\operatorname{cg} 16867657$ (-10 bp) & ELOVL2 & Blood & Pyrosequencing & 3.9 years \\
\hline & cg10501210 (+6 bp) & C1orf132 & & & \\
\hline & cg07553761 (+10 bp) & TRIM59 & & & \\
\hline & cg14361627 & KLF14 & & & \\
\hline & cg06639320 (+6 bp) & FHL2 & & & \\
\hline \multirow[t]{3}{*}{ Park et al. (57) } & cg21572772 & ELOVL2 & Blood & Pyrosequencing & 3.4 years \\
\hline & cg04208403 & ZNF423 & & & \\
\hline & cg19283806 & CCDC102B & & & \\
\hline \multirow[t]{3}{*}{ Lee et al. (58) } & cg06304190 & TTC7B & Semen & Methylation SNaPshot & 5.4 years \\
\hline & cg06979108 & NOX4 & & & \\
\hline & cg12837463 & & & & \\
\hline \multirow[t]{4}{*}{ Bekaert et al. (60) } & cg02228185 & ASPA & Blood, teeth & Pyrosequencing & 4.9 years \\
\hline & cg17861230 (-14 bp) & PDE4C & & & \\
\hline & cg16867657 (-4 bp) & ELOVL2 & & & \\
\hline & cg09809672 & EDARADD & & & \\
\hline \multirow[t]{11}{*}{ Giuliani et al. (61) } & $4 \mathrm{CpG}$ units & ELOVL2 & Cementum, dental pulp & EpiTYPER & 1.2 years $^{\mathrm{d}}$ \\
\hline & $4 \mathrm{CpG}$ units & FHL2 & & & \\
\hline & 5 CpG units & ELOVL2 & Dental pulp & & 2.3 years $^{\mathrm{d}}$ \\
\hline & 5 CpG units & FHL2 & & & \\
\hline & 3 CpG units & PENK & & & \\
\hline & $7 \mathrm{CpG}$ units & ELOVL2 & Cementum & & 2.5 years $^{\mathrm{d}}$ \\
\hline & 4 CpG units & FHL2 & & & \\
\hline & $1 \mathrm{CpG}$ units & PENK & & & \\
\hline & 3 CpG units & ELOVL2 & Dentin & & 7.1 years $^{d}$ \\
\hline & $1 \mathrm{CpG}$ units & FHL2 & & & \\
\hline & $1 \mathrm{CpG}$ units & PENK & & & \\
\hline
\end{tabular}

${ }^{a}$ Age signatures indicate the CpGs used for model construction. When CpG differs from that investigated in the BeadChip array, distance is indicated in parenthesis. + and - indicates downstream and upstream, respectively. ${ }^{\text {Accuracy }}$ is indicated by MAD from chronological age. When indicated by root-mean-square error or median of absolute difference, accuracy is marked by superscript ${ }^{\mathrm{C}}$ or $^{\mathrm{d}}$, respectively. 
and gradual loss in later life across the genome (46). After birth, average DNA methylation levels increase in the blood throughout the first year of life $(47,48)$, and after the first year, the median global DNA methylation levels are relatively stable except some regions that frequently gain methylation (47). After reaching adulthood, DNA methylation generally decreases across the genome and specifically at repetitive elements (46).

With the advent of microarray technology, more specific DNA methylation changes at certain genes or genomic regions have been reported to show high association with age (46, 49, 50) (Table 1). These age-associated CpGs have been found both within a specific tissue and across tissues, because DNA methylation profiles are highly divergent in different tissues. In earlier studies (51-53), Polycomb group protein target genes (PCGTs) known to have roles in the chromatin remodeling related with gene silencing were reported to gain methylation in blood and other tissues during aging. Bocklandt et al. (10) identified three age-associated $\mathrm{CpG}$ sites from the promoters of EDARADD, TOM1L1, and NPTX2 in saliva based on the $27 \mathrm{~K}$ BeadChip array results and built a regression model that could predict the age of an individual with an average accuracy of 5.2 years. Koch and Wagner (49) also used the 27K array results on various tissues and cells and suggested an age-predictive model composed of five CpGs (associated with the genes NPTX2, TRIM58, GRIA2, KCNQ1DN, and $B I R C 4 B P$ ), which was applicable for many tissues, but the average absolute difference between the predicted and chronological age was $\sim 11$ years. Of the studies using the 450K BeadChip array, Garagnani et al. (54) demonstrated a high association between the age and DNA methylation at three CpG sites of the genes ELOVL2, FHL2, and PENK and suggested ELOVL2 as the most promising age predictive marker in blood. A quantitative aging model built by Hannum et al. (11) used $71 \mathrm{CpG}$ sites of the $450 \mathrm{~K}$ array and showed a very high prediction accuracy with an error of 3.9 years in blood. Horvath (12) developed a multi-tissue predictor of age using 8,000 samples from the $27 \mathrm{~K}$ and $450 \mathrm{~K}$ array data sets. The model was applicable across a broad spectrum of tissues and showed a high accuracy with an error of 3.6 years.

However, the genome-wide DNA methylation analysis requires a substantial amount of DNA, time, and effort; therefore, an assay utilizing only a few $\mathrm{CpG}$ sites was expected to be more appealing to forensic investigators if it could provide accuracy comparable to that provided by the genome-wide methylation profiling. The model by Weidner et al. (13) was notable, as it was based on the pyrosequencing data of only three CpGs at the genes ITGA2B, ASPA, and $P D E 4 C$ for age prediction of blood, and the accuracy was high with an average absolute difference between the predicted and chronological age of 4.3 years. In the forensic field, Zbiec-Piekarska et al. (55) reported an age predictive model for blood using two CpGs in the ELOVL2 gene with a prediction error of 6.85 years and a mean absolute deviation
(MAD) from chronological age of 5.03 years. Later, they also demonstrated that a model composed of five CpGs of the genes ELOVL2, C1orf132, TRIM59, KLF14, and FHL2 had an improved prediction accuracy in blood with a MAD from chronological age of 3.9 years (56). Recently, Park et al. (57) also reported an age predictive model for blood using three CpGs of the genes ELOVL2, ZNF423, and CCDC102B. These CpGs were evaluated in more than 760 blood samples based on a pyrosequencing platform, and the model provided a very high prediction accuracy with a MAD from the chronological age of 3.4 years. They mentioned that the DNA methylation at KLF14 and FHL2 were significantly associated with age; however, the primer design for pyrosequencing of these genes failed. Because strong age correlation of DNA methylation at the genes ELOVL2, KLF14, and FHL2 have been repeatedly observed in many independent studies with blood, they are considered to be some of the most promising age-predictive markers for blood.

Recently, Lee et al. (58) identified age-associated CpGs for semen, which is a particularly relevant body fluid in the forensic analyses, using the $450 \mathrm{~K}$ array and subsequent methylation SNaPshot analyses. Although the model by Horvath is applicable to the age prediction with various types of tissues and cells, the age prediction values for sperm were significantly lower than the chronological age of the donors (12). The model by Lee et al. composed of three CpGs (cg06304190 in the TTC7B gene, cg06979108 in the NOX4 gene, and cg12837463) exhibited a high correlation between the predicted age and the chronological age with a MAD from chronological age of $\sim 5$ years. In addition, the region around the TTC7B gene has been reported to show age-related DNA methylation alteration in the sperm methylome of two samples collected from each individual 9-19 years apart (59), indicating $T T C 7 B$ as one of the most promising age predictive marker for semen.

Besides, Bekaert et al. (60) constructed a model with four age-associated markers suggested for blood (ASPA, PDE4C, ELOVL2, and EDARADD) and demonstrated that the model was also capable of producing highly accurate age predictions for teeth samples with a MAD from chronological age of 4.9 years. Giuliani et al. (61) also showed that the previously reported age-associated markers for blood, i. e., CpGs located in the ELOVL2, FHL2, and PENK genes (54), could be a powerful tool to predict age in teeth; however, the MAD from chronological age varied (1.2-7.1 years) depending on the part of the tooth from which DNA was extracted.

\section{Exposure-related DNA methylation changes and their potential of forensic application}

Environmental factors such as cigarette smoking and alcohol consumption may also alter DNA methylation (62). Environmental effects during early embryogenesis may induce extensive, soma-wide modifications of DNA methylation, whereas environmental effects in later life are more likely to 
induce less extensive, tissue-specific modifications of DNA methylation (25).

Cigarette smoke is considered as one of the most powerful environmental modifiers of DNA methylation (63) and is implicated in both effects during early embryogenesis and later stage of life. It may modulate DNA methylation through the mechanisms related to carcinogen-induced DNA damage and repair (64), downregulating effect of nicotine on DNMTs (65), and hypoxia (66).

Breitling et al. (63) generated DNA methylation profiles of peripheral lymphocytes from smokers, ever-smokers, and never-smokers with the use of $27 \mathrm{~K}$ BeadChip array and reported that a single locus, cg03636183 located in F2RL3 gene is differentially methylated between smokers and non-smokers. This CpG showed significantly lower methylation in smokers than non-smokers (\% methylation difference = $12 \%$ ) and has been also observed in other independent studies (67). Later, several epigenome-wide studies have been conducted using the $450 \mathrm{~K}$ BeadChip array to identify smoking-associated DNA methylation changes $(67,68)$. These studies replicated the previous findings related to F2RL3 and revealed that DNA methylation at multiple CpGs located in the $A H R R$ were also significantly lower in smokers than non-smokers. These sites (cg23576855 and cg05575921) showed smoking-related hypomethylation in the lungs and in the peripheral lymphocytes, but the percentage methylation difference between smokers and non-smokers in the lungs was much larger than in the peripheral lymphocytes $(34 \%$ vs. $17 \%$ ). Another study using more than 2,000 whole blood samples also demonstrated significantly low DNA methylation at these sites in smokers ( $\%$ methylation difference $=24 \%$ ) (69). The protein encoded by aryl hydrocarbon receptor repressor $(A H R R)$ participates in the aryl hydrocarbon receptor signaling cascade, mediating detoxification of environmental pollutants (70). Thereby, cigarette smoking decreases the AHRR DNA methylation, and related increases in the AHRR expression may remove harmful environmental chemicals such as polycyclic aromatic hydrocarbons contained in cigarette smoking (25).

However, it should be noted that such DNA methylation changes differ between tissues. Wu et al. (71) showed that a significant difference existed in the DNA methylation between the blood and saliva even from non-smokers. Therefore, potential tissue variability should also be examined to predict the effect of cigarette smoking with various tissues. Moreover, prenatal exposure to cigarette smoke induces DNA methylation change at the AHRR and CYP1A1 genes in the cord blood and placental DNA (72). However, at these sites, DNA methylation differences between the exposed and nonexposed newborns were $\leq 10 \%$, and potential DNA methylation change after that time should be investigated in various tissues.

Other studies have shown that alcohol consumption may induce DNA methylation changes in blood (73). Global DNA methylation increases in peripheral blood samples of the patients with alcoholism $(74,75)$. Early candidate gene-based investigations have generally focused on the genes related to neurotransmitter systems and reported small but significant changes associated with alcohol dependence (AD) (73). However, the loci of these studies failed to replicate previous association or reported association only in subgroups (76-78). Epigenome-wide analyses using the $27 \mathrm{~K}$ and $450 \mathrm{~K}$ BeadChip arrays reported many significant probes, which are mostly hypomethylated by alcohol consumption (79-81). Zhang et al. (79) reported that the differential methylation regions in the $\mathrm{AD}$ patients include dehydrogenases $1 \mathrm{~A}, A D H 7, A D H 3 B 2$, CYP2A13, and five loci (C8orf4, HCRTR1, FL)38379, HSA277841, and TSC2) with methylation difference of $\geq 40 \%$. Zhao et al. (80) produced DNA methylation profiles of AD-discordant siblings using the $450 \mathrm{~K}$ BeadChip array and reported 865 hypomethylated and 716 hypermethylated loci with a methylation difference of $\geq 20 \%$; the most hypomethylated CpG was located in the promoter of SSTR4 and the most hypermethylated CpG was GABRP. Philibert et al. (81) also investigated alcohol-associated methylation changes using the 450K BeadChip array, but they selected active heavy alcohol consumers entering and exiting the treatment for alcohol use disorders and compared them to community controls. In this report, significant changes within individuals were not detected at four weeks following the treatment entry, but 8626 CpGs were found to be differentially methylated between the current heavy drinkers and controls. However, methylation differences at these loci were generally $\leq 10 \%$. Moreover, almost all the alcohol users (85\%) were daily smokers, and the cg09935388 at GFI1 gene with the largest value of $15 \%$ has been reported in a number of smoking-related studies, thereby suggesting a possible confounding effect at this site (73). In contrast to smoking, numerous limitations were found both in terms of quality and number of studies on alcohol (73). The primary weakness is the lack of replicated findings, which may attribute to the difference in the study design and population investigated. In addition, the effect size is relatively small with the largest methylation difference frequently at $\leq 10 \%$ (73).

In an effort to establish tools to aid diagnosis and monitor treatment response by environmental exposures, Endo et al. (82) developed an assay to measure DNA methylation at smoking and alcohol consumption-associated CpGs using the MethyLight method that utilizes fluorescence-based real-time PCR technology. They selected cg23576855 in the AHRR gene as smoking and cg02583484 in the HNRNPA1 gene as alcohol consumption biomarkers and tested their specificity in 33 blood samples from healthy donors. The methylation rate at cg23576855 was significantly different between the current and never smokers (never smokers: $72.0 \pm 9.5$, past smokers: $65.8 \pm 8.0$, current smokers: $44.4 \pm 15.1$ ), and the AUC for the DNA methylation rate was 0.955 for current smokers. The cg02583484 was selected from the study by Philibert et al. (81), and the methylation rate at this site could differentiate among never and habitual alcohol consumers (never drinkers: 
$58.5 \pm 13.2$, habitual alcohol drinkers: $48.1 \pm 9.1$ ). In the ROC analysis, the AUC for the DNA methylation rate was 0.746 for habitual alcohol consumers. Although the DNA methylation rates of $A H R R$ and HNRNPA1 did not correlate with the frequency of smoking and alcohol consumption (82), DNA methylation at these sites seems to have the potential to monitor the lifestyle of the blood sample donors. Further analyses with more various types of tissues would be also helpful. Once the specificity and the effect size of selected markers are confirmed to be suitable as biomarkers, cigarette smoking and alcohol consumption-associated DNA methylation changes could be used for the prediction of individual's life style, and will also be useful in reducing the number of potential suspects.

\section{Assay design for DNA methylation measurement in forensic evidence samples}

Because DNA methylation patterns are erased by PCR amplification, an extra step is needed to convert DNA methylation information into readily assayable DNA sequence information (83). Above all, the chemical treatment of the DNA with sodium bisulfite gives rise to methylation-specific sequence variants, which can be mapped and quantified by various epigenome-wide technologies and locus-specific analysis.

Assay design means the development of locus-specific analysis for the detection of single or a few genes that are already established as predictive biomarkers. Therefore, selecting relevant markers to allow the detection of desired characteristics is essential. In general, relevant markers can be selected from studies employing epigenome-wide technologies. Whole genome bisulfite sequencing, bisulfite microarray, and enrichment methods are the current most useful and popular approaches (83), and among them, bisulfite microarray is the most public and easily accessible. The latest 450K BeadChip array allows high-resolution, genome-wide DNA methylation profiling of human samples, covering $99 \%$ of all the RefSeq genes and $\sim 450,000$ CpGs overall (84). In addition, its low cost allows the profiling of a large number of samples, thereby now enormous data can be browsed and downloaded from the public databases, e. g., Gene Expression Omnibus (GEO) database of the US National Center for Biotechnology Information (83).

Once the markers to be included in the assay are determined, locus-specific analysis can be designed using several approaches. Although an approach based on the use of MSRE has been reported in the forensic body fluid identification, sequencing-based technologies such as direct bisulfite pyrosequencing or alternatives including methylationsensitive single nucleotide primer extension-based analysis are more suitable for reliably assessing DNA methylation level in terms of reproducibility and resolution. Direct bisulfite pyrosequencing has been most widely used in forensic field as well as in other fields. This approach has been reported to provide considerable reproducibility with $\geq 10$ ng of DNA $(56,85)$. Tost and Gut $(86)$ reported high reproducibility of the pyrosequencing method with differences in the methylation results of only $2 \%$ in the case of the same PCR reaction and $5 \%$ in the case of different bisulfite treatments and/or separate PCR reactions. However, because this assay does not allow multiplexing of markers, a lot of time, cost, and efforts may be required when multiple markers are analyzed in a large number of samples.

The second most popular approach used in the forensic field is the methylation-sensitive single nucleotide primer extensionbased approach, generally called methylation SNaPshot. This method enables simultaneous analysis of multiple markers by designing multiplex methylation SNaPshot, and methylation levels at multiple $\mathrm{CpG}$ sites can conveniently be observed at a glance in the electropherogram. In addition, it uses the same workflow used in the forensic SNP analysis except that bisulfite-converted DNA is used as a template, thus special training is not required for an assay. However, the signal strength between the fluorescence dyes in the electropherograms is different, and therefore this method is most favored when detecting "on-off" signal rather than measuring hyper or hypomethylation signal. As such, this method has been consistently used for assaying markers for forensic body fluid identification (Fig. 1) $(44,87)$. The sensitivity varied from 0.01 to $0.5 \mathrm{ng}$ of bisulfite-converted DNA (0.05 to $10 \mathrm{ng}$ of genomic DNA) depending on the marker $(44,85,87)$, indicating the importance of primer design and sequence analyzed in the assay. Alternatives such as MethyLight and Epityper methods employing real time-PCR or MALDI-TOF mass spectrometry could also be used for assay design, but they do not provide 1 bp resolution $(61,82)$.

Because each method has different limitations, the assay should be designed in consideration of markers to be analyzed. For example, bisulfite pyrosequencing can be more suitable for the analysis of $\mathrm{CpG}$ islands than methylation SNaPshot, because the SBE reaction of methylation SNaPshot may be easily affected by CpGs adjacent to the target CpG site. Moreover, if the assay needs precise quantitative measurement of DNA methylation such as age-prediction analysis, the determination of the lowest DNA concentration that guarantees reproducibility will be much more crucial during assay validation, because the methylation rate represents the frequency of methylation at a given $\mathrm{CpG}$ site across the population of cells taken from a sample vial and would be affected by the number of DNA strands included.

\section{CONCLUDING REMARKS}

Recent advances in epigenetics have suggested that DNA methylation markers can provide more informative layers to the evidence in forensic analysis. DNA methylation profiling not only can provide information about tissue origin of evidence sample, but also can provide information about the 

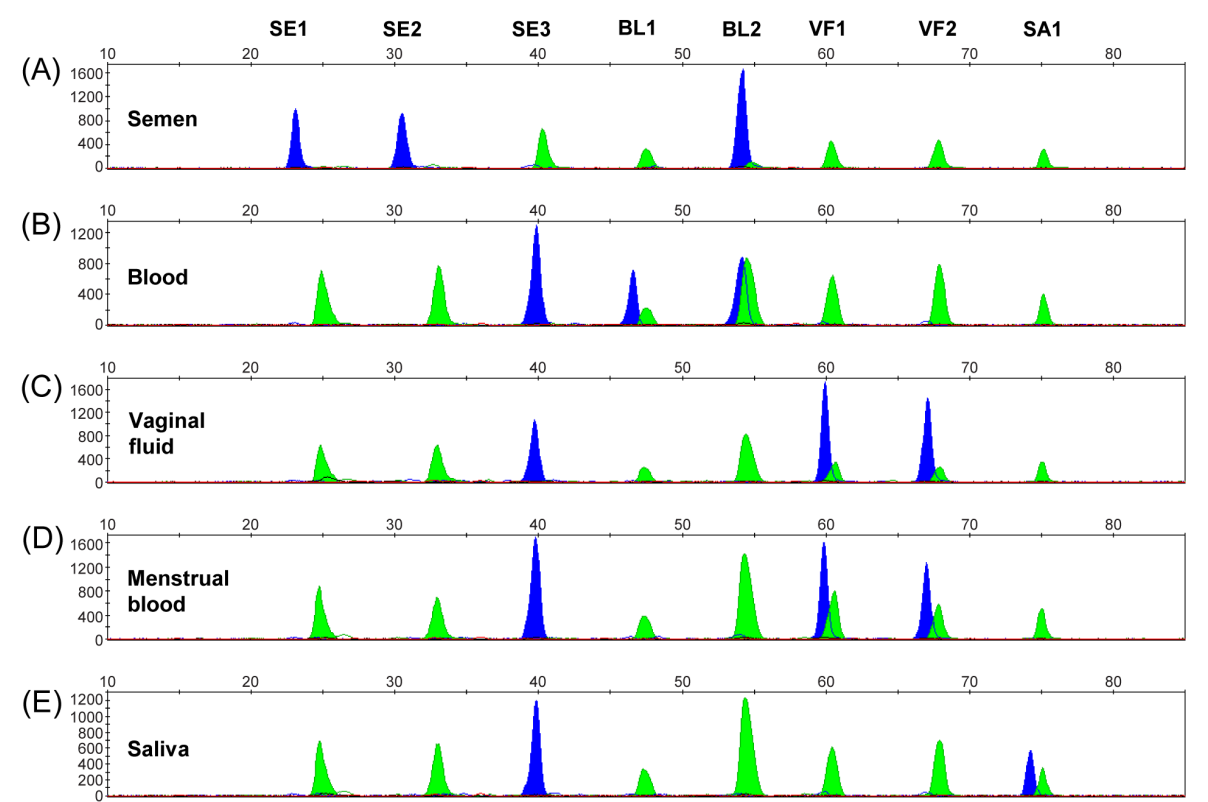

Fig. 1. Representative electropherograms of body fluid identification using multiplex methylation SNaPshot. (A) Semen, (B) blood, (C) vaginal fluid, (D) menstrual blood, and (E) saliva. SE1, SE2, SE3, BL1, BL2, VF1, VF2, and SA1 represent cg17610929, cg26763284, cg17621389, cg06379435, cg01543184, cg09765089-231d, cg26079753-7d, and cg09652652-2d, respectively. In a DNA methylation profile produced by the multiplex methylation SNaPshot, a green peak represents the nucleotide $A$ as an unmethylation signal and a blue peak represents the nucleotide $G$ as a methylation signal. Reprinted from Lee et al. (44) with kind permission from Elsevier. age and history of environmental exposure of an individual. The ability to infer the unknown sample donor's age or lifestyle such as smoking and alcohol consumption habits can guide police investigations in cases without known suspects, thereby allowing forensic use of DNA methylation for investigation, not in the courtroom. In contrast, body fluid identification that allows predicting the activity leading to the deposition of the evidence material can play an important role to evaluate the evidence at the activity level in the courtroom. However, because cell lineage and tissue of origin are major determinants of DNA methylation, the identification of the type of tissues or body fluids is an essential prerequisite to obtain investigative leads such as predicted age and lifestyle using DNA methylation profiling of evidence materials. Therefore, the identification of CpG markers for more types of tissues and body fluids and following assay development to detect them need to be encouraged. More and more DNA methylation markers are being suggested as age-specific, smoking-specific, and alcohol consumption-specific markers; however, some of them failed to replicate previous findings and require further validation with various types of tissues and cells. In addition, because forensic evidence materials often possess only a scarce amount of DNA, determining the lowest DNA concentration that guarantees reliable quantitative results will be important. Moreover, some methylation markers show methylation changes of a mere of $10 \%$ or less; therefore, careful choice should be made in assay design so that the analysis platform employed could be able to detect the methylation change at least equivalent to the effect size of the marker. Nevertheless, DNA methylation has great potential to provide additional useful information to current forensic DNA profiling. In addition, DNA methylation profiling procedures fit well with the current forensic workflow, and accordingly could be easily integrated into forensic standardized procedures. Therefore, in the near future, forensic investigations should significantly improve with the continued advances in epigenetics to allow the extraction of more investigative leads from forensic evident materials.

\section{ACKNOWLEDGEMENTS}

This study was supported by the Bio \& Medical Technology Development Program of the National Research Foundation of Korea (NRF) funded by the Korean government (NRF-2014M 3A9E1069992).

\section{REFERENCES}

1. Kayser M (2015) Forensic DNA Phenotyping: Predicting human appearance from crime scene material for investigative purposes. Forensic Sci Int Genet 18, 33-48

2. Santos F, Machado H and Silva S (2013) Forensic DNA databases in European countries: is size linked to performance? Life Sci Soc Policy 9, 12

3. European Network of Forensic Science Institutes DNA Working Group, Group DW. Report on Criminal Cases in Europe solved by ILS (DNA Mass Testing). http://www. enfsi.eu/sites/default/files/documents/enfsi_report_on_crim inal cases in europe solved by ils 0.pdf, 2006

4. Kayser M and Schneider PM (2009) DDNA-based prediction of human externally visible characteristics in forensics: motivations, scientific challenges, and ethical considerations. Forensic Sci Int Genet 3, 154-161 
5. Kayser $M$ and de Knijff $P$ (2011) Improving human forensics through advances in genetics, genomics and molecular biology. Nat Rev Genet 12, 179-192

6. Sulem P, Gudbjartsson DF, Stacey SN et al (2007) Genetic determinants of hair, eye and skin pigmentation in Europeans. Nat Genet 39, 1443-1452

7. Walsh S, Lindenbergh A, Zuniga SB et al (2011) Developmental validation of the IrisPlex system: determination of blue and brown iris colour for forensic intelligence. Forensic Sci Int Genet 5, 464-471

8. Walsh S, Chaitanya L, Clarisse L et al (2014) Developmental validation of the HIrisPlex system: DNA-based eye and hair colour prediction for forensic and anthropological usage. Forensic Sci Int Genet 9, 150-161

9. Zubakov D, Liu F, van Zelm MC et al (2010) Estimating human age from T-cell DNA rearrangements. Curr Biol 20, R970-R971

10. Bocklandt S, Lin W, Sehl ME et al (2011) Epigenetic predictor of age. PLoS ONE 6, e14821

11. Hannum G, Guinney J, Zhao L et al (2013) Genome-wide methylation profiles reveal quantitative views of human aging rates. Mol Cell 49, 359-367

12. Horvath S (2013) DNA methylation age of human tissues and cell types. Genome Biol 14, R115

13. Weidner Cl, Lin Q, Koch CM et al (2014) Aging of blood can be tracked by DNA methylation changes at just three CpG sites. Genome Biol 15, R24

14. Jackson G (2013) The impact of commercialization on the evaluation of DNA evidence. Front Genet 4, 227

15. McKenna L (2013) Understanding DNA results within the case context: importance of the alternative proposition. Front Genet 4, 242

16. Gunn P, Walsh S and Roux C (2014) The nucleic acid revolution continues, will forensic biology become forensic molecular biology? Front Genet 5, 44

17. Sijen T (2015) Molecular approaches for forensic cell type identification: On mRNA, miRNA, DNA methylation and microbial markers. Forensic Sci Int Genet 18, 21-32

18. An JH, Shin KJ, Yang WI and Lee HY (2012) Body fluid identification in forensics. BMB Rep 45, 545-553

19. Virkler K and Lednev IK (2009) Analysis of body fluids for forensic purposes: from laboratory testing to non-destructive rapid confirmatory identification at a crime scene. Forensic Sci Int 188, 1-17

20. Schübeler D (2015) Function and information content of DNA methylation. Nature 517, 321-326

21. Bestor TH (1990) DNA methylation: evolution of a bacterial immune function into a regulator of gene expression and genome structure in higher eukaryotes. Philos Trans R Soc Lond B Biol Sci 326, 179-187

22. Jones PA (2012) Functions of DNA methylation: islands, start sites, gene bodies and beyond. Nat Rev Genet 13, 484-492

23. Chen T, Ueda Y, Dodge JE, Wang Z and Li E (2003) Establishment and maintenance of genomic methylation patterns in mouse embryonic stem cells by Dnmt3a and Dnmt3b. Mol Cell Biol 23, 5594-5605

24. Tahiliani M, Koh KP, Shen $Y$ et al (2009) Conversion of 5-methylcytosine to 5-hydroxymethylcytosine in mammalian DNA by MLL partner TET1. Science 324, 930-935
25. Lee KW and Pausova Z (2013) Cigarette smoking and DNA methylation. Front Genet 4, 132

26. Rakyan VK, Down TA, Balding DJ and Beck S (2011) Epigenome-wide association studies for common human diseases. Nat Rev Genet 12, 529-541

27. Rakyan VK, Hildmann T, Novik KL et al (2004) DNA methylation profiling of the human major histocompatibility complex: a pilot study for the human epigenome project. PLoS Biol 2, e405

28. Frigola J, Song J, Stirzaker C, Hinshelwood RA, Peinado $\mathrm{MA}$ and Clark SJ (2006) Epigenetic remodeling in colorectal cancer results in coordinate gene suppression across an entire chromosome band. Nature Genet 38, 540-549

29. Irizarry RA, Ladd-Acosta C, Wen B et al (2009) The human colon cancer methylome shows similar hypo- and hypermethylation at conserved tissue-specific $\mathrm{CpG}$ island shores. Nature Genet 41, 178-186

30. Straussman R, Nejman D, Roberts D et al (2009) Developmental programming of $\mathrm{CpG}$ island methylation profiles in the human genome. Nat Struct Mol Biol 16, 564-571

31. Martin GM (2005) Epigenetic drift in aging identical twins. Proc Natl Acad Sci U S A 102, 10413-10414

32. Bjornsson HT, Sigurdsson Ml, Fallin MD et al (2008) Intra-individual change over time in DNA methylation with familial clustering. JAMA 299, 2877-2883

33. Kaminsky ZA, Tang T, Wang SC et al (2009) DNA methylation profiles in monozygotic and dizygotic twins. Nat Genet 41, 240-245

34. Bell JT, Pai AA, Pickrell JK et al (2011) DNA methylation patterns associate with genetic and gene expression variation in HapMap cell lines. Genome Biol 12, R10

35. Numata S, Ye T, Hyde TM et al (2012) DNA methylation signatures in development and aging of the human prefrontal cortex. Am J Hum Genet 90, 260-272

36. Byun HM, Siegmund KD, Pan F et al (2009) Epigenetic profiling of somatic tissues from human autopsy specimens identifies tissue- and individual-specific DNA methylation patterns. Hum Mol Genet 18, 4808-4817

37. Davies MN, Volta M, Pidsley R et al (2012) Functional annotation of the human brain methylome identifies tissue-specific epigenetic variation across brain and blood. Genome Biol 13, R43

38. Ziller MJ, Gu H, Müller F et al (2013) Charting a dynamic DNA methylation landscape of the human genome. Nature 500, 477-481

39. Jiang R, Jones MJ, Chen E et al (2015) Discordance of DNA methylation variance between two accessible human tissues. Sci Rep 5, 8257

40. Frumkin D, Wasserstrom A, Budowle B and Davidson A (2011) DNA methylation-based forensic tissue identification. Forensic Sci Int Genet 5, 517-524

41. Lee HY, Park MJ, Choi A, An JH, Yang WI and Shin KJ (2012) Potential forensic application of DNA methylation profiling to body fluid identification. Int J Legal Med 126, 55-62

42. Madi T, Balamurugan K, Bombardi R, Duncan G and McCord B (2012) The determination of tissue-specific DNA methylation patterns in forensic biofluids using 
bisulfite modification and pyrosequencing. Electrophoresis 33, 1736-1745

43. Park JL, Kwon OH, Kim JH et al (2014) Identification of body fluid-specific DNA methylation markers for use in forensic science. Forensic Sci Int Genet 13, 147-153

44. Lee HY, An JH, Jung SE et al (2015) Genome-wide methylation profiling and a multiplex construction for the identification of body fluids using epigenetic markers. Forensic Sci Int Genet 17, 17-24

45. Forat S, Huettel B, Reinhardt R et al (2016) Methylation Markers for the Identification of Body Fluids and Tissues from Forensic Trace Evidence. PLoS One 11, e0147973

46. Jones MJ, Goodman SJ and Kobor MS (2015) DNA methylation and healthy human aging. Aging Cell 14, 924-932

47. Martino DJ, Tulic MK, Gordon L et al (2011) Evidence for age-related and individual-specific changes in DNA methylation profile of mononuclear cells during early immune development in humans. Epigenetics 6, 10851094

48. Jenkins TG, Aston KI, Cairns BR and Carrell DT (2013) Paternal aging and associated intraindividual alterations of global sperm 5-methylcytosine and 5-hydroxymethylcytosine levels. Fertil Steril 100, 945-951

49. Koch C and Wagner W (2011) Epigenetic-aging-signature to determine age in different tissues. Aging 3, 1018-1027

50. Bell JT, Tsai PC, Yang TP et al (2012) Epigenome-wide scans identify differentially methylated regions for age and age-related phenotypes in a healthy ageing population. PLoS Genet 8, e1002629

51. Viré E, Brenner C, Deplus R et al (2006) The Polycomb group protein $\mathrm{EZH} 2$ directly controls DNA methylation. Nature 439, 871-874

52. Teschendorff AE, Menon U, Gentry-Maharaj A et al (2010) Age-dependent DNA methylation of genes that are suppressed in stem cells is a hallmark of cancer. Genome Res 20, 440-446

53. Heyn H, Li N, Ferreira HJ et al (2012) Distinct DNA methylomes of newborns and centenarians. Proc Natl Acad Sci U S A 109, 10522-10527

54. Garagnani P, Bacalini MG, Pirazzini C et al (2012) Methylation of ELOVL2 gene as a new epigenetic marker of age. Aging Cell 11, 1132-1134

55. Zbieć-Piekarska R, Spólnicka M, Kupiec T et al (2015) Examination of DNA methylation status of the ELOVL2 marker may be useful for human age prediction in forensic science. Forensic Sci Int Genet 14, 161-167

56. Zbieć-Piekarska R, Spólnicka M, Kupiec T et al (2015) Development of a forensically useful age prediction method based on DNA methylation analysis. Forensic Sci Int Genet 17, 173-179

57. Park JL, Kim JH, Seo E et al (2016) Identification and evaluation of age-correlated DNA methylation markers for forensic use. Forensic Sci Int Genet 23, 64-70

58. Lee HY, Jung SE, Oh YN, Choi A, Yang WI and Shin KJ (2015) Epigenetic age signatures in the forensically relevant body fluid of semen: a preliminary study. Forensic Sci Int Genet 19, 28-34

59. Jenkins TG, Aston KI, Pflueger C, Cairns BR and Carrell DT (2014) Age-associated sperm DNA methylation alterations: possible implications in offspring disease susceptibility. PLoS Genet 10, e1004458

60. Bekaert B, Kamalandua A, Zapico SC, Van de Voorde W and Decorte R (2015) Improved age determination of blood and teeth samples using a selected set of DNA methylation markers. Epigenetics 10, 922-930

61. Giuliani C, Cilli E, Bacalini MG et al (2016) Inferring chronological age from DNA methylation patterns of human teeth. Am J Phys Anthropol 159, 585-595

62. Terry MB, Delgado-Cruzata L, Vin-Raviv N, Wu HC and Santella RM (2011) DNA methylation in white blood cells: association with risk factors in epidemiologic studies. Epigenetics 6, 828-837

63. Breitling LP, Yang R, Korn B, Burwinkel B and Brenner $H$ (2011) Tobacco-smoking-related differential DNA methylation: $27 \mathrm{~K}$ discovery and replication. Am J Hum Genet $88,450-457$

64. Huang J, Okuka M, Lu W et al (2012) Telomere shortening and DNA damage of embryonic stem cells induced by cigarette smoke. Reprod Toxicol 35, 89-95

65. Satta R, Maloku E, Zhubi A et al (2008) Nicotine decreases DNA methyltransferase 1 expression and glutamic acid decarboxylase 67 promoter methylation in GABAergic interneurons. Proc Natl Acad Sci U S A 105, 16356-16361

66. Liu Q, Liu L, Zhao $Y$ et al (2011) Hypoxia induces genomic DNA demethylation through the activation of HIF-1alpha and transcriptional upregulation of MAT2A in hepatoma cells. Mol Cancer Ther 10, 1113-1123

67. Shenker NS, Polidoro S, Van Veldhoven K et al (2012) Epigenome-wide association study in the European Prospective Investigation into Cancer and Nutrition (EPIC-Turin) identifies novel genetic loci associated with smoking. Hum Mol Genet 22, 843-851

68. Monick MM, Beach SR, Plume J et al (2012) Coordinated changes in AHRR methylation in lymphoblasts and pulmonary macrophages from smokers. Am J Med Genet B Neuropsychiatr Genet 159B, 141-151

69. Zeilinger S, Kühnel B, Klopp N et al (2013) Tobacco smoking leads to extensive genome-wide changes in DNA methylation. PLoS One 8, e63812

70. Opitz CA, Litzenburger UM, Sahm F et al (2011) An endogenous tumour-promoting ligand of the human aryl hydrocarbon receptor. Nature 478, 197-203

71. Wu HC, Wang Q, Chung WK et al (2014) Correlation of DNA methylation levels in blood and saliva DNA in young girls of the LEGACY Girls study. Epigenetics 9, 929-933

72. Joubert BR, Haberg SE, Nilsen RM et al (2012) 450K epigenome-wide scan identifies differential DNA methylation in newborns related to maternal smoking during pregnancy. Environ Health Perspect 120, 1425-1431

73. Andersen AM, Dogan MV, Beach SR and Philibert RA (2015) Current and Future Prospects for Epigenetic Biomarkers of Substance Use Disorders. Genes 6, 991-1022

74. Bonsch D, Lenz B, Reulbach U, Kornhuber J and Bleich S (2004) Homocysteine associated genomic DNA hypermethylation in patients with chronic alcoholism. J Neural Transm 111, 1611-1616

75. Semmler A, Heese P, Stoffel-Wagner B et al (2015) 
Alcohol abuse and cigarette smoking are associated with global DNA hypermethylation: Results from the german investigation on neurobiology in alcoholism (GINA). Alcohol 49, 97-101

76. Nieratschker V, Grosshans M, Frank J et al (2014) Epigenetic alteration of the dopamine transporter gene in alcohol-dependent patients is associated with age. Addict Biol 19, 305-311

77. Jasiewicz A, Rubis B, Samochowiec J et al (2015) DAT1 methylation changes in alcohol-dependent individuals vs. controls. J Psychiatr Res 64, 130-133

78. Philibert RA, Gunter TD, Beach SR, Brody GH and Madan A (2008) MAOA methylation is associated with nicotine and alcohol dependence in women. Am J Med Genet B Neuropsychiatr Genet 147B, 565-570

79. Zhang $\mathrm{H}$, Wang $\mathrm{F}$, Kranzler $\mathrm{HR}$, Zhao $\mathrm{H}$ and Gelernter J (2013) Profiling of childhood adversity-associated DNA methylation changes in alcoholic patients and healthy controls. PLoS ONE 8, e65648

80. Zhao R, Zhang R, Li W et al (2013) Genome-wide DNA methylation patterns in discordant sib pairs with alcohol dependence. Asia Pac Psychiatry 5, 39-50

81. Philibert RA, Penaluna B, White T et al (2014) A pilot examination of the genome-wide DNA methylation signatures of subjects entering and exiting short-term alcohol dependence treatment programs. Epigenetics 9, 1212-1219

82. Endo K, Li J, Nakanishi M et al (2015) Establishment of the MethyLight Assay for Assessing Aging, Cigarette Smoking, and Alcohol Consumption. Biomed Res Int 2015, 451981

83. Bock C (2012) Analysing and interpreting DNA methylation data. Nat Rev Genet 13, 705-719

84. Sandoval J, Heyn H, Moran S et al (2011) Validation of a DNA methylation microarray for $450,000 \mathrm{CpG}$ sites in the human genome. Epigenetics 6, 692-702

85. Silva DS, Antunes J, Balamurugan K, Duncan G, Alho CS and McCord B (2016) Developmental validation studies of epigenetic DNA methylation markers for the detection of blood, semen and saliva samples. Forensic Sci Int Genet $23,55-63$

86. Tost J and Gut IG (2007) DNA methylation analysis by pyrosequencing. Nat Protoc 2, 2265-2275

87. An JH, Choi A, Shin KJ, Yang WI and Lee HY (2013) DNA methylation-specific multiplex assays for body fluid identification. Int J Legal Med 127, 35-43 